\title{
Imaging Mouse Prostate Gland by 3 Tesla Clinical MRI System
}

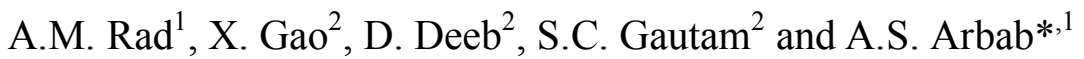 \\ Department of ${ }^{1}$ Radiology and ${ }^{2}$ Surgery, Henry Ford Hospital, Detroit, Michigan, USA
}

\begin{abstract}
In vivo detection of prostate tumor in animal model will facilitate the investigations that deal with the efficacy of different treatment strategies in different experimental settings. Recently higher field strength dedicated animal MRI system has been used successfully to detect mouse prostate glands and its lesions, however, usefulness of clinical system has not been utilized to its fullest extent. In this short communication we show the advantages and disadvantages of different in vivo imaging parameters of MRI to acquire images of the mouse prostate gland using clinical strength MRI systems.
\end{abstract}

\section{INTRODUCTION}

According to American Cancer Society, prostate cancer is the most frequently diagnosed malignant neoplasm among American men and remains the second leading cause of cancer-related deaths [1] and (www.cancer.org). The American Cancer Society estimates that there will be about 186,320 new cases of prostate cancer in the United States in 2008. Investigators in the field of prostate cancer therapy and research mostly use subcutaneous tumor models to predict therapeutic responses in different pre-clinical treatment strategies; more recently orthotopic mouse prostate cancer models with human cancer cells implanted in the mouse prostate or transgenic models, such as TRAMP (Transgenic Adenocarcinoma of Mouse Prostate) mice, which develop spontaneous tumors, have been used to study chemopreventive treatment efficacy [2-4]. Extracting and weighing the prostate at different time points is the current method to determine the efficacy of treatment. Ability to non-invasively detemine the size and volume of a mouse's prostate gland as well as tumor will help investigators in the diagnosis and evaluation of the efficacy of different treatment strategies in prostate cancer. Investigators have employed magnetic resonance imaging (MRI) to assess therapeutic efficacy in preclinical studies but only very few reached the necessary high resolution which would allow a good delineation of prostate glands and its tumor, however, all these MRI studies that acquired high resolution images were performed on higher field strength animal systems $(4.7 \mathrm{~T}$ or $7.0 \mathrm{~T})[5,6]$. Comparing available in vivo imaging modalities, magnetic resonance imaging (MRI) is thought to be the best choice because of its high resolution, availability for animal system (high field animal MRI) and small animal coils that can be used in a clinical system. Moreover, different MRI parameters and sequences can be utilized to identify different structures based on tissue characteristics of prostate gland and tumor.

\footnotetext{
*Address correspondence to this author at the Cellular and Molecular Imaging Laboratory, Department of Radiology, Henry Ford Hospital, 1 Ford Place, 2F, Box 82, Detroit, MI 48202, USA; Tel: 313-874-4435; Fax: 313874-4494; E-mail: saali@rad.hfh.edu
}

Compared to human, prostate gland in mouse is different in shape and size and does not remain fixed. Mouse prostate is multi-lobed (two anterior, two dorsolateral and one ventral) which surrounds urethra (Fig. 1) and moves along with urinary bladder. Therefore, it is a challenging task to identify the mouse prostate by any in vivo imaging method. The purpose of this short communication is to show the utility of clinical MRI system to identify normal and tumor bearing mouse prostate gland, to discuss the effective pulse sequences, and to show the MRI of prostate gland on three different imaging planes.

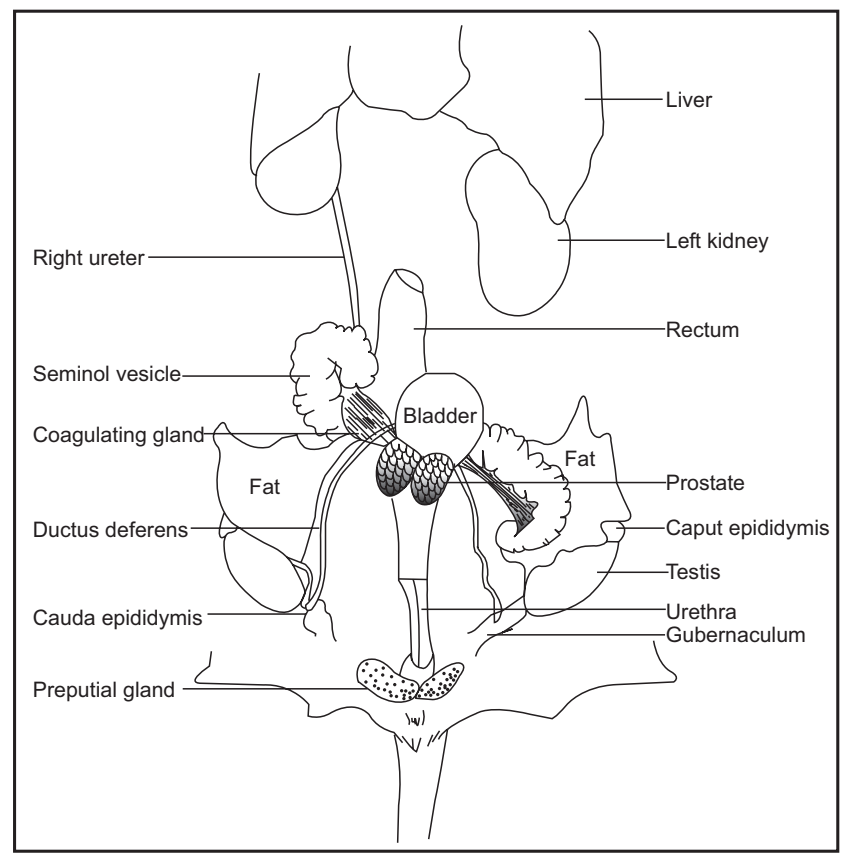

Fig. (1). Schematic illustration of mouse urogenital systems adapted from "The Anatomy of the Laboratory Mouse by Margaret J. Cook, M.R.C. Laboratory Animals Centre, Carshalton, Surrey, England Academic Press, 1965" with permission. Copyright Elsevier. 


\section{MATERIALS AND METHODS}

\section{Animals and Tumor}

Three controls and two tumor bearing male nude mice were included in this feasibility MRI study. The protocols and the use of animals were approved by institutional animal care and user committee (IACUC). Tumor was produced by implanting $5 \mu$ of cells suspension $\left(1 \times 10^{6} \mathrm{C} 4-2 \mathrm{~B}\right.$ human prostate cancer cells in matrigel) orthotopically in exposed prostate gland through a surgical incision at lower abdomen. Animals were anesthetized by ketamine-xylazine injection and cleaned with betadine. Injection was proceeded under microscope by a microinjector. Fourteen days after tumor implantation, tumor bearing animals underwent MRI scan session.

\section{MRI Coils}

Small animal Litzcage RF volume coils (50 $\mathrm{mm}$ diameter $\mathrm{x} 108 \mathrm{~mm}$ RF rung length and $38 \mathrm{~mm}$ diameter x $64 \mathrm{~mm} \mathrm{RF}$ rung length) for a single channel, tuned to ${ }^{1} \mathrm{H}$ at $128 \mathrm{MHz}$ (3 Tesla) were purchased from Doty Scientific, Inc, (Columbia, SC). The coils were synchronized with a clinical 3 tesla MRI systems (Signa Excite, GE health). All pulse sequences used were clinically relevant and supplied by the vendor (GE health).

\section{In Vivo MRI Studies by 3 Tesla}

An appropriate state of anesthesia was obtained with isoflurane (3\% for induction, $0.7 \%$ to $1.5 \%$ for maintenance mixed with $\mathrm{O}_{2}$ ). MRI was obtained with a 3 Tesla clinical system (Signa Excite, GE health) using either $50 \mathrm{~mm}$ diameter $x 108 \mathrm{~mm}$ RF rung length or $38 \mathrm{~mm}$ diameter $\mathrm{x} 64 \mathrm{~mm}$ RF rung length small animal imaging coil (Litzcage small animal imaging system, Doty Scientific Inc, Columbia, SC). After positioning using a triplanar FLASH sequence, T1, T2and fat suppression T2-weighted images were obtained. T1weighted images were obtained using following sequence; $\mathrm{TR} / \mathrm{TE}=300 / 10 \mathrm{~ms}, 128 \times 128$ matrix, $13-15$ slices, $0.9 \mathrm{~mm}$ thick, 30 to $40 \mathrm{~mm}$ field of view (FOV), number of excitation $(\mathrm{NEX})=2-4$. T2-weighted images were obtained using standard two-dimensional Fourier transformation (2DFT) multislice (13-15) multiecho (2-4 echoes) MRI. A series of 2-4 sets of images (13-15 slices for each set) were obtained using TEs of 10 to $70 \mathrm{msec}$ and a TR of $1500-4500 \mathrm{~ms}$. The images were acquired using 25 to $40 \mathrm{~mm} \mathrm{FOV}, 0.7$ to 0.9 $\mathrm{mm}$ slice thickness, $128 \times 128$ matrix, and NEX $=2-4$. Fat saturation T2-weighted images were also obtained using similar imaging parameters.

\section{RESULTS}

\section{How to Identify Prostate Gland in the Mouse}

It is difficult to identify the prostate gland if testes are set as a reference point. The testes $(\mathrm{t})$ move from within the abdominal cavity to a position outside the body cavity due to muscle contraction, room and body temperature. To identify prostate gland either from axial, sagittal or coronal images, urinary bladder should be the reference point and the prostate gland is located anatomically inferior and posterior to the bladder, encircling urethra. If bladder is not set as a reference point, preputial gland can be mistaken for prostate gland (arrow heads on Figs. 2, 4 and 5).

\section{Ability of MRI to Detect Prostate Gland}

Application of 3 tesla clinical system using small animal coils is illustrated in Fig. (2). Coronal views (D,E) illustrated prostate gland and surrounding urogenital organs in the mouse (compare with Fig. 1). To identify the prostate gland along with other structures of urogenital system, images along coronal plane should be acquired.

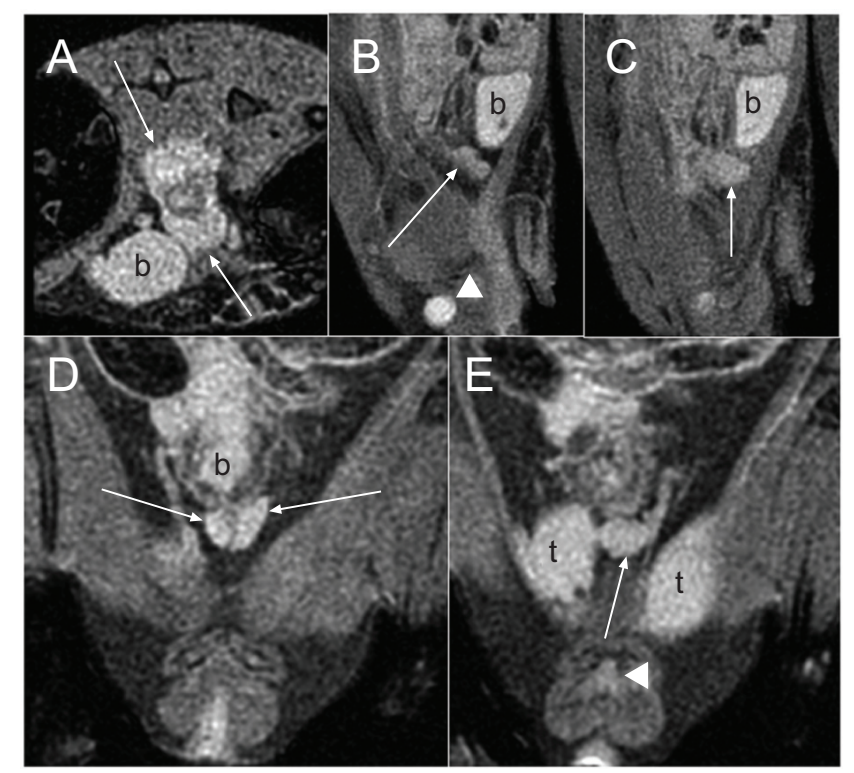

Fig. (2). Identification of mouse prostate gland on MRI. The images were obtained by a 3 tesla MRI system using 50x108 mm Litzcage volume coil by Doty Scientific, using following imaging parameters: fast spin echo FATSAT T2-weighted sequence, TR/TE $=2000 / 13-15 \mathrm{~ms}, 25$ to $30 \mathrm{~mm}$ FOV, $128 \times 128$ matrices, $\mathrm{NEX}=3$, slice thickness 0.7 to $0.9 \mathrm{~mm}$. (A) axial, (B, C) sagittal, (D, E) coronal sections are showing prostate gland (white arrows). $\mathrm{b}=$ urinary bladder, $\mathrm{t}=$ testes. White arrows indicate the prostate gland. Arrow heads $(\mathrm{B}, \mathrm{E})$ indicate the preputial gland.

\section{Advantage of T2-Weighted Images}

Use of proper image sequence is essential to identify the mouse prostate gland and its demarcation from surrounding organs. Fig. (3) shows the advantage of T2-weighted image (T2WI) over conventional T1-weighted image (T1WI). The signal intensity of the prostate gland on T1WI was similar to that of the surrounding muscle tissues and on T1WI the prostate gland could not be distinguished confidently from surrounding muscles or tissues compared to that of images obtained with T2-weighted sequence (white arrows on 3B). On T2WI the prostate gland showed higher signal intensity compared to that of surrounding muscle tissues and could easily be separated. The signal intensity of the prostate gland was comparable to that of the content of urinary bladder.

\section{Advantage of FAT Saturation Pulse and Long TE}

Lower abdominal cavity of mouse is full of fat (Fig. 1), which surrounds urogenital systems. To identify and demarcate the mouse prostate gland, a fat saturation pulse should be applied during MRI acquisition. Fig. (4) shows the advantage of using fat saturation pulse to identify the prostate gland from surrounding tissues (compare 4A vs. 4B). Fig. (4) 
also demonstrates the advantage of long TE. Images obtained with long TE (C, $20 \mathrm{~ms}$ vs. D, $40 \mathrm{~ms}$ ) showed better demarcation of prostate gland from its surrounding muscles.

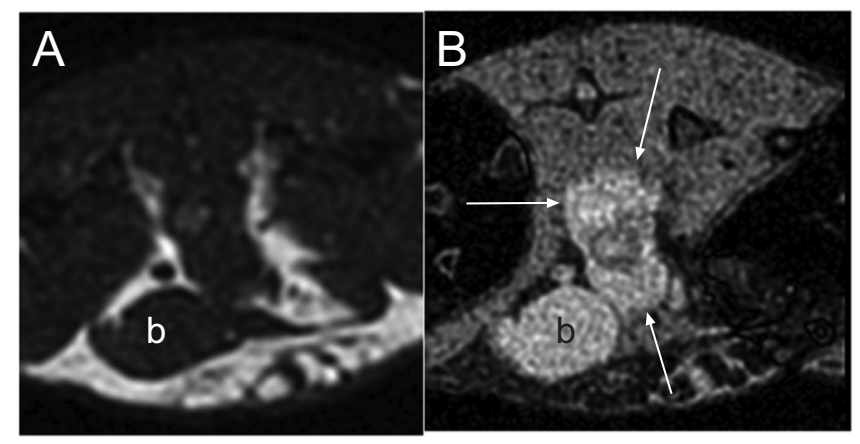

Fig. (3). Advantage of T2-weighted over T1-weighted images. (A) Axial T1-weighted image obtained using following parameters: $\mathrm{TR} / \mathrm{TE}=300 / 10 \mathrm{~ms}, 40 \times 40 \mathrm{~mm}$ FOV, $128 \times 128$ matrices, $\mathrm{NEX}=2$. (B) Axial FSE fatsat T2-weighted image obtained using following parameters: TR/TE $=1800 / 13 \mathrm{~ms}, 25 \times 25 \mathrm{~mm}$ FOV, $128 \times 128 \mathrm{ma}-$ trices, $\mathrm{NEX}=4$, slice thickness $0.7 \mathrm{~mm}$. Note the clear demarcation of prostate gland on $\mathrm{T} 2$-weighted image (white arrows). $\mathrm{b}=$ urinary bladder.

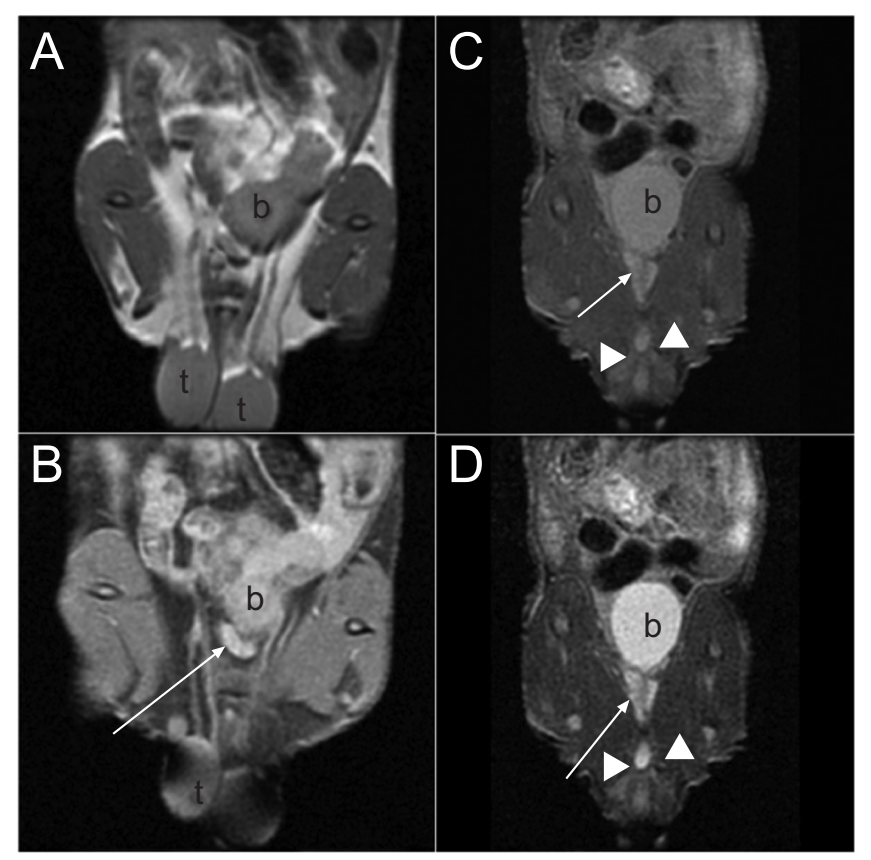

Fig. (4). Advantages of FATSAT and longer TE for T2weighted images. (A) Axial FSE T2-weighted image obtained using following parameters: TR/TE $=3850 / 12 \mathrm{~ms}, 30 \times 30 \mathrm{~mm}$ FOV, $128 \times 128$ matrices, NEX $=3$, slice thickness $1.2 \mathrm{~mm}$. (B) FATSAT axial FSE T2-weighted image obtained at the same plane using following parameters: TR/TE $=3850 / 12 \mathrm{~ms}, 30 \times 30 \mathrm{~mm}$ FOV, $128 \times 128$ matrices, NEX $=3$, slice thickness $1.2 \mathrm{~mm}$. Note the clear demarcation of the prostate gland on FATSAT T2-weighted image (white arrow). (C) FATSAT T2-weighted image obtained with short TE $(20 \mathrm{~ms})$ and (D) longer TE $(40 \mathrm{~ms})$ using similar parameters indicated above in $\mathrm{A}$ and $\mathrm{B}$. $\mathrm{b}=$ urinary bladder, $\mathrm{t}=$ testes. Arrow heads (C,D) indicate preputial gland.

\section{MRI of Mouse Prostate Gland Tumor}

Fig. (5) demonstrates orthotopically implanted human prostate cancer cells in the left dorsolateral lobe of the prostate gland (white arrows). The images showed the advantage of fatsat T2WI with long TE to identify the tumor from the surrounding tissues and the prostate gland. The central high signal intensity could be due to glandular structure of the prostate cancer as well as possible central necrosis. The tumor was clearly identified and demarcated on all image planes, which was easily distinguishable from urinary bladder and normal prostate lobe (right dorsolateral lobe).

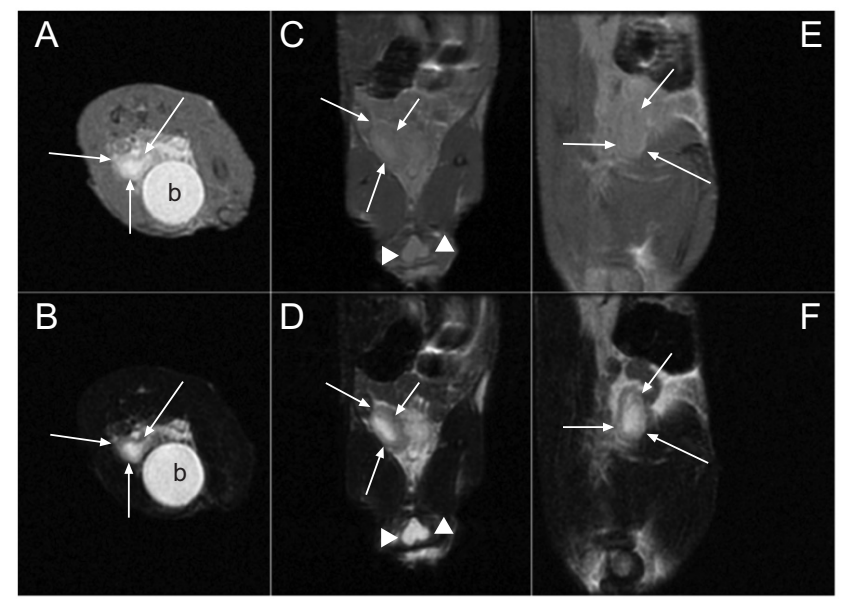

Fig. (5). Detection of implanted tumor in the left dorsolateral lobe of prostate by in vivo MRI. Tumors are shown in axial (A, B), coronal (C, D) and sagittal (E, F) planes. FATSAT T2weighted images were obtained with short TE (12-13 ms, A, C and E) and long TE (69-73 ms, B, D and F). Note the better demarcation of tumor on images with long TE (arrows). $b=$ urinary bladder, $\mathrm{t}=$ testes. White arrows indicate the tumor in prostate gland. Arrow heads (D) indicate preputial gland.

\section{Measurement of Tumor and the Prostate Gland}

Clinical strength MRI has limitation to produce high resolution images and the calculated tumor or prostate gland volume could be limited due to partial volume effect. However, the measurement of the tumor (length and width) as well as the total length and width of the extracted tumor bearing prostate gland were comparable to the in vivo measurement, which indicated the validity of our study using clinical strength MRI (Fig. 6).

\section{DISCUSSION}

The results of this study show the utility of clinical strength MRI systems coupled with small animal coils in acquiring relatively high resolution MR images of small animal prostate gland and associated tumor. Use of different image sequences and fat suppression techniques indicate the robustness of clinical systems in applying clinically relevant MRI sequences without increasing the image acquisition time. The time to acquire different sets of images in our study ranges from 5 minutes to maximum of 15 minutes, which is considerably shorter or comparable with the imaging time in recently reported publication of MRI of the mouse prostate gland using dedicated small animals systems 


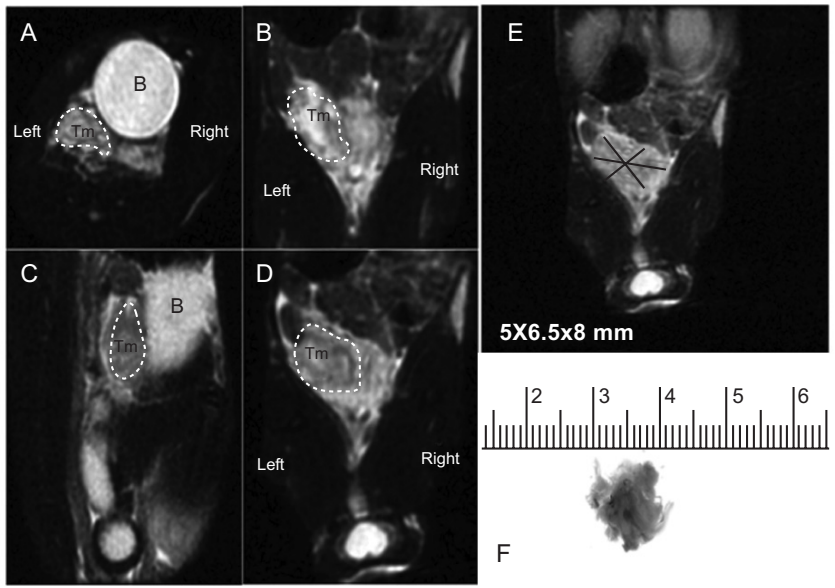

Fig. (6). Measurement of tumor and prostate gland. Tumor is outlined on axial, sagittal and coronal plans (A-D) indicating accurate demacation of tumor from normal prostate gland. Length of prostate gland including tumor $(8 \mathrm{~mm})$, length $(6.5 \mathrm{~mm})$ and width $(5 \mathrm{~mm})$ of the implanted tumor alone are shown $(\mathbf{E})$, which are comparable to the tumor bearing extracted gland $(\mathbf{F})$.

$[5,6]$. Although the spatial resolution and the signal to noise of the images reported in this work are reasonably comparable with the same sort of images acquired with the animal systems, the image thickness, which is more than double than what it is possible to obtain with a higher gradient strength on animal instruments, could be a limitation for accurate measurement of the volume of the prostate gland considering the small size of the prostate. However, with proper setting and acquisition averages (increasing acquisition time), the in-plan image slice thickness could be as low as 0.4 to $0.5 \mathrm{~mm}$. On the other hand, measurement of tumor volume may not be difficult due to rapid growth of the implanted tumor, which was comparable with the extracted tumor in our study.

Proper identification of the prostate gland in small animal model is crucial to determine the effect of different treatment strategies. Use of clinical strength MRI systems will be an advantage due to availability of the systems in most of the academic institutes as well as in hospitals. However, selection of optimal MRI sequence is essential during acquisition of images. There has been a report of the mouse prostate gland imaging using 1.5 tesla clinical MRI systems; however, compared to the images of the current study the prostate glands and its tumors were not clearly visualized [7]. Our study also indicates the utility of fat suppression technique and longer TE in acquiring T2-weighted images, which showed both the prostate gland and the implanted tumor clearly. The similar image sequences can be used to determine the efficacy of treatment in a longitudinal study. The importance of this feasibility study on a clinical system is that more investigators will have access to a clinical instrument than to a dedicated animal system and similar study can easily be performed.

In vivo MR images have the ability to determine the size and volume of the prostate gland before and after treatment, which can replace the current method of extraction and weighing of whole prostate gland after treatment. In vivo MRI will decrease the number of animals needed to complete experimental studies to determine treatment efficacy. Each animal can be used as its own control. User-friendly image analysis software will allow not only to determine the size and volume of the gland/tumor after treatment but also to characterize early treatment changes by analyzing different MRI parameters such as $\mathrm{T} 2$ or $\mathrm{T} 2 *$ relaxation values, apparent diffusion coefficient (ADC), perfusion and permeability, etc. Moreover, the use of clinical MRI system together with clinically relevant image sequences will make the findings clinically applicable.

\section{CONCLUSIONS}

Clinical strength MRI system can be used effectively to image mouse prostate gland and its tumor.

\section{ACKNOWLEDGEMENTS}

Part of the study was supported by grants from National Institutes of Health (NIH). R01CA122031 and R21NS585892.

\section{REFERENCES}

[1] Jemal A, Siegel R, Ward E, et al. Cancer statistics, 2008. CA Cancer J Clin 2008; 58(2): 71-96.

[2] Gupta S, Ahmad N, Marengo SR, MacLennan GT, Greenberg NM, Mukhtar H. Chemoprevention of prostate carcinogenesis by alphadifluoromethylornithine in TRAMP mice. Cancer Res 2000; 60(18): 5125-33

[3] Gupta S, Hastak K, Ahmad N, Lewin JS, Mukhtar H. Inhibition of prostate carcinogenesis in TRAMP mice by oral infusion of green tea polyphenols. Proc Natl Acad Sci USA 2001; 98(18): 10350-5.

[4] Fricke ST, Rodriguez O, Vanmeter J, et al. In vivo magnetic resonance volumetric and spectroscopic analysis of mouse prostate cancer models. Prostate 2006; 66(7): 708-17.

[5] Degrassi A, Russo M, Scanziani E, et al. Magnetic resonance imaging and histopathological characterization of prostate tumors in TRAMP mice as model for pre-clinical trials. Prostate 2007; 67(4): 396-404.

[6] Nastiuk KL, Liu H, Hamamura M, Muftuler LT, Nalcioglu O, Krolewski JJ. In vivo MRI volumetric measurement of prostate regression and growth in mice. BMC Urol 2007; 7: 12.

[7] Gupta S, Adhami VM, Subbarayan M, et al. Suppression of prostate carcinogenesis by dietary supplementation of celecoxib in transgenic adenocarcinoma of the mouse prostate model. Cancer Res 2004; 64(9): 3334-43.

(C) Rad et al.; Licensee Bentham Open.

This is an open access article distributed under the terms of the Creative Commons Attribution License (http://creativecommons.org/licenses/by/2.5/), which permits unrestrictive use, distribution, and reproduction in any medium, provided the original work is properly cited. 